\title{
The good and bad sides of exosomes: pre- metastatic niche formation, cancer biomarker and therapy carriers
}

\author{
Isabella Panfoli', Maurizio Bruschi ${ }^{2}$ \\ 'Dipartimento di Farmacia-DIFAR, Università di Genova, Genoa, Italy. \\ ${ }^{2}$ Laboratory of Molecular Nephrology, IRCCS Istituto Giannina Gaslini, Genoa 16147, Italy.
}

Correspondence to: Prof. Isabella Panfoli, Dipartimento di Farmacia-DIFAR, Università di Genova, Scuola di Scienze Mediche e Farmaceutiche, DIFAR, Viale Benedetto XV, 3, Genoa 16132, Italy. E-mail: panfoli@difar.unige.it

How to cite this article: Panfoli I, Bruschi M. The good and bad sides of exosomes: pre-metastatic niche formation, cancer biomarker and therapy carriers. J Cancer Metastasis Treat2020;6:35. http://dx.doi.org/10.20517/2394-4722.2020.50

Received: 25 May 2020 First Decision: 18 Jun 2020 Revised: 2 Jul 2020 Accepted: 21 Jul 2020 Published: 27 Sep 2020

Academic Editor: Robert J. Griffin Copy Editor: Cai-Hong Wang Production Editor: Jing Yu

\begin{abstract}
Exosomes, nanovesicles of endocytic origin, are secreted by most cell types; cancer cells representing no exception. Exosomes facilitate intercellular communication as they deliver diverse proteins, mRNA, miRNA and lipids. In this review, we discuss how exosomes represent one of the main risks associated with cancer but also one of the most promising new tools to fight it. Exosomes appear to function as signalling molecules between the tumour microenvironment, i.e., the complex of both cancer and stromal cells, and the rest of the body. Cancerderived exosomes have been shown to drive the initiation and progression of metastasis, by transporting their cargoes to target tissues. In this respect, exosomes are implicated in cancer progression, dissemination and therapy resistance. However, exosomes are also emerging as a key tool in precision medicine, pivotal for cancer liquid biopsy in early diagnosis and for assessing when there is a recurrence. Profiling exosomal cancer-derived nucleic acids by ultrasensitive next-generation sequencing along with mapping the protein profile utilizing highthroughput proteomics will allow earlier cancer detection, therapeutic stratification and monitoring of response to therapy. Exosomes are also a promising new tool for cancer immunotherapy. Clinically utilizing exosomes for these applications in cancer diagnosis and therapeutics will be the next challenge.
\end{abstract}

Keywords: Biomarkers, cancer, exosomes, immunotherapy, liquid biopsy, precision medicine, proteomics

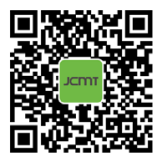




\section{INTRODUCTION}

Exosomes (Exo) are nanovesicles secreted by virtually any cell and found in all human body fluids $s^{[1-4]}$. They play important roles in intercellular communication both in physiology and disease $\mathrm{e}^{[4-6]}$. After being released into the extracellular space, Exo can enter the circulation and ultimately be taken up by recipient cells to which they can convey their protein, nucleic acid and lipid cargo in turn modulating their activity ${ }^{[7,8]}$. By proteomic and biochemical analyses, we have reported a novel characteristic of human Exo, i.e., they can consume oxygen to aerobically synthesize $\mathrm{ATP}^{[9-11]}$. Proteomic and statistical analyses of both human mesenchymal stem cell (MSC) and urinary Exo showed enrichment of proteins related to aerobic metabolism, including the redox complexes I, IV, and V in their membranes ${ }^{[9-11]}$. Such aerobic metabolic capacity appears consistent with the Exo need to maintain their cup-shaped structure and functionality in the face of a prolonged permanence in the circulation ${ }^{[10-12]}$ and with their ability to rescue bioenergetics of damaged cells ${ }^{[13,14]}$.

Research in the field of cancer is benefiting from the growing understanding of the biogenesis and functions of Exo released from cancer cells ${ }^{[15-18]}$. However, the role of Exo in the pathophysiology of cancer is multifaceted ${ }^{[19]}$. Cancer cell-derived Exo have long been known to be involved in processes such as cancer progression, metastasis, immune escape, angiogenesis and therapy resistance ${ }^{[20,21]}$. Exo function as signalling molecules between the tumour microenvironment, i.e., the complex of both cancer and stromal cells, and the rest of the body ${ }^{[16]}$. Tumour-derived Exo can drive the metastatic process by targeting specific organs setting a pre-metastatic environment ${ }^{[17]}$. The tumour environment reacts by amplifying oncogenic pathways in which Exo signalling is proposed to play a role ${ }^{[22]}$.

Nonetheless, Exo also contain biomarkers, and can be considered biomarkers themselves ${ }^{[2,24]}$. For example, urinary Exo deriving from every cell of the urinary tract and kidney, may represent a promising reservoir of cancer biomarkers to assess disease progression in urologic cancers ${ }^{[25,26]}$. Exo can be noninvasively isolated from human urine to detect biomarkers, with promising applications in patient stratification, monitoring of therapy response or use as vehicles for therapeutic delivery ${ }^{[27]}$.

Thanks to high throughput mass spectrometry techniques, it can be envisioned that Exo will help in earlier diagnosis, directing therapeutic targets and assessing therapy resistance upon detection of signature cargo biomarker biomolecules ${ }^{[1,16]}$. In the landscape of precision medicine, cancer liquid biopsies, i.e., the analysis of circulating cell-free tumour DNA and tumour cells (CTC ${ }^{[28]}$, can exploit examining Exo signatures, when they are derived from biological fluids ${ }^{[29]}$. Exo can also potentially be used as tumour-targeting vehicles for cancer ${ }^{[17,18,21]}$. There is also the exciting possibility of engineering the content of Exo and targeting them to tissues of interest ${ }^{[30,31]}$.

This review focuses on the dual role of Exo in cancer. The good side of Exo is their clinical potential. The ability of Exo to mediate cell-to-cell communication as stable carriers of molecular messages can be exploited for cancer diagnosis and therapy, with novel exciting perspectives of translation to the clinical setting ${ }^{[16,32]}$. The bad side of Exo is the long-recognized ability of their cargo to modify the cancer microenvironment promoting cancer progression and metastasis by recruiting target cells, as is the case for melanoma ${ }^{[33]}$.

\section{EXOSOMES}

Exo, a subset of extracellular vesicles (EVs) of endocytic origin, actively shed from multivesicular bodies (MVBs) which carry proteins, RNAs (mRNA, miRNA, transfer RNA, ribosomal RNA, nucleolar RNA, and noncoding RNA) and DNA fragments ${ }^{[1,3,7,34]}$. Exo biogenesis involves the formation of early endosomal vesicles, that develop into late endosomes undergoing inward budding forming intraluminal vesicles 
(ILVs) in $\mathrm{MVBs}^{[6,35]}$. Exo acquire their luminal and membrane contents through both direct and stochastic sorting $^{[6]}$. If nascent ILVs are not degraded by fusion with lysosomes, they fuse with the plasma membrane and are released into the extracellular space ${ }^{[36]}$. Once in the circulation, Exo are directed to other cells via an unclear mechanism, probably involving members of the tetraspannin family (CD9, CD63 and CD81), where they are taken up by the target tissues through membrane fusion, endocytosis, or receptor-ligand interaction $^{[2,34,37,38]}$.

Exo isolation involves a variety of techniques ${ }^{[39,40]}$. Several commercial exosome isolation kits are in use, although their ability to yield pure and functional Exo is uncertain ${ }^{[41]}$. To date, differential ultracentrifugation is considered the gold standard, and it is much preferred when pure Exo are required. Low-speed centrifugation subsequent to polyethylene glycol precipitation has been utilized to enrich Exo from large sample volumes, but there is the possibility of polymer contamination ${ }^{[42]}$. The other Exo isolation methods are size-based, antibody capture-based, microfluidics-based techniques or precipitation by altering Exo solubility with water-excluding polymers such as polyethylene glycol, all of which do not require high-speed centrifugation ${ }^{[40]}$. Fluidic techniques such as exosome total isolation chip, can sort Exo from a heterogeneous population of EVs based on their size, with the advantage of allowing the handling of small sample volumes ${ }^{[43]}$. Label-free efficient separation of Exo from human blood has been reported, using an integrated continuous-flow acoustofluidic device ${ }^{[4]}$. This last automated isolation technique is useful in case of biohazard, and for integration with downstream Exo analysis systems ${ }^{[4]}$. Notably, most novel isolation methods are limited by the requirement of sophisticated instrumentation or costly reagents. Therefore, notwithstanding their promising potential, a concern about the clinical applications of Exo appears to be the standardization of isolation techniques.

\section{THE GOOD SIDE OF EXOSOMES}

Exo are emerging as promising and sensitive cancer biomarkers for disease diagnosis ${ }^{[2,23,24,26]}$. Once released from cancer cells, Exo enter the circulation and are transported in biological fluids carrying their functional cargoes $^{[1]}$. Profiling Exo cancer-derived nucleic acids by ultrasensitive next-generation sequencing and proteins by large-scale high-throughput proteomics could allow early cancer detection, therapeutic stratification and response to therapy monitoring ${ }^{[17,18,38,45,46]}$.

Liquid biopsy has drawn attention as a minimally invasive and cost-effective method for sampling of genetic, proteomic and metabolic material from different types of cancer ${ }^{[47]}$. While traditional cancer liquid biopsies utilize cell free DNA or $\mathrm{CTC}^{[48]}$, Exo are emerging as a novel tool in the field of precision medicine in the early diagnostic and recurrence assessment applications ${ }^{[24,49]}$. This has proven true especially for urologic tumours ${ }^{[29,50]}$. The advantage of Exo over existing approaches is their stability in body fluids allowing the specific cancer-derived proteins and nucleic acids to be preserved ${ }^{[1,4]}$. For example, a cell membrane-anchored proteoglycan, glypican-1 (GPC1), overexpressed in a variety of cancers such as breast and pancreatic cancer, was also identified in the circulating Exo from the serum of pancreatic cancerbearing patients. Detection of $\mathrm{GPC}^{+}{ }^{+}$Exo in the serum can be utilized to distinguish healthy subjects from pancreatic cancer patients with very high specificity and sensitivity, findings that can then be correlated with survival rates ${ }^{[51]}$. Considering its cited role in the progression of human pancreatic cancer, miR-301a-3p was proposed as a biomarker in the diagnosis of this type of cancer ${ }^{[52]}$. An Exo-specific "melanoma signature" with prognostic and therapeutic potential has been proposed ${ }^{[33]}$.

Exo can also become stable and reliable engineered nanocarriers ${ }^{[23]}$. In fact, the benefits of the use of Exo as therapeutic agents include their biocompatibility and ability to deliver their content to specific target cells, an unfavourable feature per se, that can however be of advantage. The characteristic exosomal membrane stability, which is enriched in cholesterol, sphingomyelin and ceramide, along with its tumourtargeting capacity, has suggested the use of Exo in anticancer therapy delivering therapeutic miRNAs 
and proteins or as drug delivery system for cancer therapy ${ }^{[53]}$. Drug delivery with Exo has been utilized for the treatment of breast, pancreatic, lung, and prostate cancers and glioblastoma ${ }^{[54]}$. Drug loading into Exo can be accomplished by both active and passive encapsulation approaches ${ }^{[5,56]}$. Passive loading can be accomplished by simple incubation of the Exo with the drug or miRNAs to let them diffuse along the concentration gradient or treating cells with a drug, and using Exo secreted by these donor cells. Active encapsulation can be performed by freeze-thawing cycles, electroporation of the Exo membranes, or sonication in the presence of the drug ${ }^{[56]}$. Sonication can be also used to encapsulate proteins. Freezethawing method has also been utilized to fuse Exo with liposomes, to develop exosome-liposome hybrids carrying specific proteins ${ }^{[57]}$. By manipulating the shedding process of Exo, these can also be engineered using viral vectors, such as retroviruses and adenoviruses to engineer the parent cells to secrete modified Exo, or alternatively, to directly manipulate the content Exo following secretion ${ }^{[30,56]}$. The use of Exo also shows significant safety and low toxicity benefits as compared to cell-based therapies, which bear the risk of activation of a host immune response. By contrast, allogenic Exo elicit lower immune response ${ }^{[30]}$. The use of MSC-derived Exo for delivery of anticancer therapy was shown to hold more promise ${ }^{[58]}$ than the use of MSC themselves ${ }^{[59]}$. The fact that MSC Exo carry an aerobic metabolic ability ${ }^{[11]}$ may also be taken into closer consideration.

Exo are also a promising new tool of cancer immunotherapy ${ }^{[15,60]}$. In lieu of dendritic cell (DC)-based cancer immunotherapy, still in its infancy due to problems associated with culture and storage, the use of DCderived Exo to generate immune responses against tumours, has been proposed ${ }^{[31]}$. DC-derived Exo possess immunostimulatory properties thanks to the expression of integrins, ICAM-1 and MHC class II and class I molecules as well. Exo stability would maintain the DC-derived Exo cancer antigen composition, including surface MHC-peptide complexes and the CD80 and CD86 costimulatory molecules, thus triggering the desired cancer antigen-specific immune response ${ }^{[31]}$. DC-derived Exo-based phase I and II clinical trials have demonstrated their ability to target cancer sites ${ }^{[61]}$.

By genetic modification of the parental cell, Exo can be loaded with a variety of biological molecules, such as miRNAs and proteins. Exo surface modification by addition of ligands or proteins to the Exo surface can direct them to target cancer cells ${ }^{[32]}$. In this respect, the features that allow Exo to reach their targets (i.e., ligands expressed onto their surface) allow their positive applications. Targeting ligands on the surface of Exo can also be engineered. For example, HEK293T cells were engineered to express the protein Lamp2b, fused to a fragment of interleukin 3 in their Exo membrane, and it was found that these Exo loaded with imatinib or with a specific siRNA were able to target cancer cells and inhibit their growth in vitro and in $v i v o^{[62]}$. Exo released by macrophages, loaded with paclitaxel (PTX) increased the cytotoxicity of PTX by about 50-fold. In vivo, those Exo co-localized with lung metastases causing a significant inhibition of their growth $^{[63]}$.

The hallmark of the double nature of Exo is their ability to deliver miRNA, small noncoding RNAs that target mRNAs and change their expression. Exo can be manipulated to have them deliver anti-miRNAs, or tumour-suppressing miRNAs for cancer treatment. It was shown that endothelial cells can transfer exogenous miRNA to cancer cells via Exo ${ }^{[64]}$.

\section{THE BAD SIDE OF EXOSOMES}

Cancer cells secrete more Exo than healthy cells ${ }^{[65]}$. Moreover, their contents appear to be tailored to mediate cancer dissemination ${ }^{[8,16,17,23]}$. Being potent signalling mediators, Exo shed from cancer cells act in facilitating cancer initiation and progression ${ }^{[20,21]}$. Exo are also secreted by cells in the tumour microenvironment (TME) such as stromal and immune cells ${ }^{[2,66]}$. TME is the complex setting of the interplay between cells and signalling events, of which Exo along with cytokines and chemokines are key players $^{[s]}$. In particular, Exo can precondition the TME setting the pre-metastatic niche, which increases 


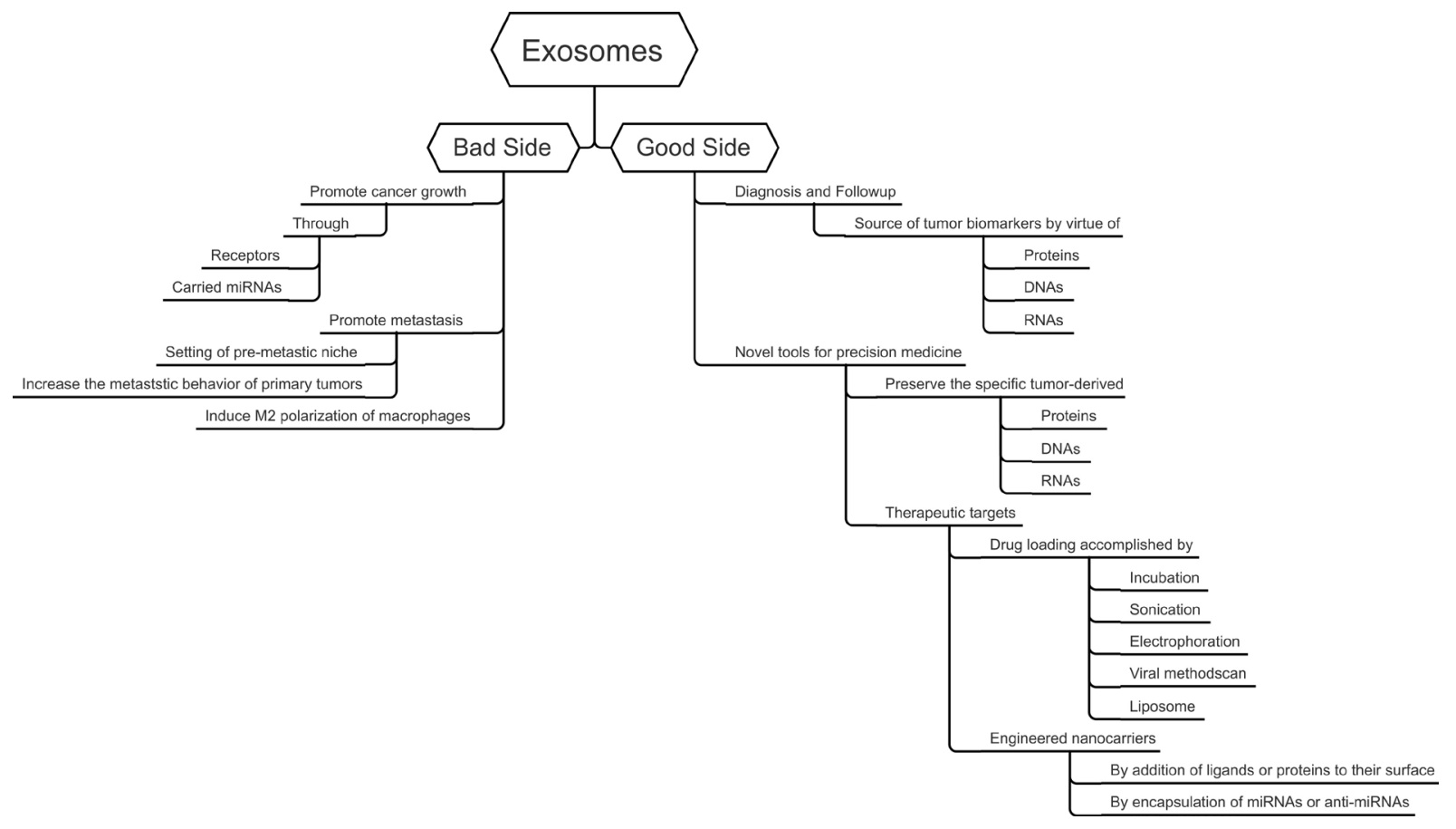

Figure 1. Schematic graphical representation of the "good" and "bad" sides of exosomes

metastatic success ${ }^{[16]}$. Evidence suggests that the contents of Exo modulate immune cell activity, enabling immune surveillance and treatment evasion. For example, Exo were shown to express programmed death-1 $(\mathrm{PD}-1)$, which interacts with programmed death-ligand 1 (PD-L1 $)^{[61]}$. Upregulation of the expression of $\mathrm{PD}-\mathrm{L} 1$ receptor on $\mathrm{T}$ cells mediates $\mathrm{PD}-\mathrm{L} 1$-dependent immune evasion, by actively suppressing the function of CD8. It was shown that exosomes expressing PD-L1 shed form metastatic melanomas helping the tumour to evade immune surveillance. Assessment of exosomal PD-L1 content has been proposed to stratify patients for therapy with anti-PD-1 antibodies, a promising treatment for metastatic melanoma ${ }^{[6]}$. While remaining a poorly understood process, metastasis is the cause of most cancer-related deaths with miRNA transfer to endothelial cells, mediated by Exo, facilitating this metastatic process ${ }^{[16]}$.

Exosomal mRNA and proteins from prostate cancer cells were shown to modulate the prostatic TME ${ }^{[2,67]}$. The formation of melanoma lung metastases is preceded by the recruitment of bone marrow progenitors primed through receptor tyrosine kinase MET activation by Exo ${ }^{[33]}$. Pancreatic cancer cell-derived Exo can induce stellate cells to recruit macrophage subpopulations, establishing a pre-metastatic environment inside the liver ${ }^{[17,33]}$. Pancreatic cancer cell Exo promote metastasis by inducing the M2 polarization of macrophages through activation of the PTEN/PI3Kg pathway ${ }^{[52]}$. It was shown that Exo miR301a-3p overexpression is associated with poor survival ${ }^{[52]}$.

Given that Exo have "good" and "bad" roles (see Schematic in Figure 1), a prerequisite for the successful implementation of their use for cancer therapy requires rigorous isolation, and characterisation. In an attempt to support the standardisation of Extracellular vesicles (EV) in research and clinical applications, an international consortium was established, named "EV-TRACK" (http://evtrack.org). EV-TRACK is a knowledgebase intended to gather and centralize reports on EV biology and methodology ${ }^{[68]}$.

\section{CONCLUSION}

Exo are nano-sized vesicles secreted from living cells into all body fluids. They bear a dual role in cancer biology, being supportive of cancer progression, by setting the tumour metastatic niche, but are also 
carriers of cancer cell-specific protein and nucleic acid contents. Exo are viewed as promising tools for cancer diagnosis and therapy. We highlight how current research is exploiting both these utilities and the strategies used to enhance the potential of Exo in the treatment of cancer. If understood in more detail, the identification of cancer-specific Exo would become vital in early diagnosis and assessing response to therapeutic intervention(s) when monitoring cancer progression and its treatment. The unique stability, biocompatibility, homing ability and low immunogenicity of Exo, can also be exploited as a delivery system for therapeutic agents since Exo can also be loaded with a variety of molecules. As our understanding of Exo biology and the profiling of their protein and RNA cargoes as potential biomarkers is expanded, the ability to engineer content of Exo opens new possibilities for their use as selective carriers of therapeutic miRNAs and/or drug carriers to target tumour cells. It is foreseeable that the next years will witness the challenging clinical applications of Exo in cancer diagnosis and therapeutics.

\section{DECLARATIONS}

\section{Authors' contributions}

Made substantial contributions to the conception and design of this study and wrote the manuscript: Panfoli I

Revised this work substantially: Bruschi M

\section{Availability of data and materials}

Not applicable.

\section{Financial support and sponsorship}

None.

\section{Conflicts of interest}

All authors declared that there are no conflicts of interest.

\section{Ethical approval and consent to participate}

Not applicable.

\section{Consent for publication}

Not applicable.

\section{Copyright}

(c) The Author(s) 2020.

\section{REFERENCES}

1. Rashed MH, Bayraktar E, Helal GK, Abd-Ellah MF, Amero P, et al. Exosomes: from garbage bins to promising therapeutic targets. Int J Mol Sci 2017; 18:538.

2. Mathivanan S, Ji H, Simpson RJ. Exosomes: extracellular organelles important in intercellular communication. J Proteomics 2010;73:1907-20.

3. Raposo G, Stoorvogel W. Extracellular vesicles: exosomes, microvesicles, and friends. J Cell Biol 2013;200:373-83.

4. Record M, Carayon K, Poirot M, Silvente-Poirot S. Exosomes as new vesicular lipid transporters involved in cell-cell communication and various pathophysiologies. Biochim Biophys Acta 2014;1841:108-20.

5. Bang C, Thum T. Exosomes: new players in cell-cell communication. Int J Biochem Cell Biol 2012;44:2060-4.

6. Vlassov AV, Magdaleno S, Setterquist R, Conrad R. Exosomes: current knowledge of their composition, biological functions, and diagnostic and therapeutic potentials. Biochim Biophys Acta 2012;1820:940-8.

7. Mathivanan S, Fahner CJ, Reid GE, Simpson RJ. ExoCarta 2012: database of exosomal proteins, RNA and lipids. Nucleic Acids Res 2012;40:D1241-4.

8. Tian W, Liu S, Li B. Potential role of exosomes in cancer metastasis. Biomed Res Int 2019;2019:4649705.

9. Bruschi M, Ravera S, Santucci L, Candiano G, Bartolucci M, et al. The human urinary exosome as a potential metabolic effector cargo. 
Expert Rev Proteomics 2015;12:425-32.

10. Bruschi M, Santucci L, Ravera S, Candiano G, Bartolucci M, et al. Human urinary exosome proteome unveils its aerobic respiratory ability. J Proteomics 2016;136:25-34.

11. Panfoli I, Ravera S, Podestà M, Cossu C, Santucci L, et al. Exosomes from human mesenchymal stem cells conduct aerobic metabolism in term and preterm newborn infants. FASEB J 2016;30:1416-24.

12. Bruschi M, Santucci L, Ravera S, Bartolucci M, Petretto A, Calzia D, et al. Metabolic signature of microvesicles from umbilical cord mesenchymal stem cells of preterm and term infants. Proteomics Clin Appl 2018;12:1700082.

13. Arslan F, Lai RC, Smeets MB, Akeroyd L, Choo A, et al. Mesenchymal stem cell-derived exosomes increase ATP levels, decrease oxidative stress and activate PI3K/Akt pathway to enhance myocardial viability and prevent adverse remodeling after myocardial ischemia/reperfusion injury. Stem Cell Res 2013;10:301-12.

14. Islam MN, Das SR, Emin MT, Wei M, Sun L, et al. Mitochondrial transfer from bone-marrow-derived stromal cells to pulmonary alveoli protects against acute lung injury. Nat Med 2012;18:759-65.

15. Chaput N, Théry C. Exosomes: immune properties and potential clinical implementations. Semin Immunopathol 2011;33:419-40.

16. Li I, Nabet BY. Exosomes in the tumor microenvironment as mediators of cancer therapy resistance. Mol Cancer 2019;18:32.

17. Wu M, Wang G, Hu W, Yao Y, Yu XF. Emerging roles and therapeutic value of exosomes in cancer metastasis. Mol Cancer 2019;18:53.

18. Li W, Li C, Zhou T, Liu X, Liu X, et al. Role of exosomal proteins in cancer diagnosis. Mol Cancer 2017;16:145.

19. Kosaka N, Yoshioka Y, Hagiwara K, Tominaga N, Katsuda T, et al. Trash or Treasure: extracellular microRNAs and cell-to-cell communication. Front Genet 2013;4:173.

20. Dutta S, Warshall C, Bandyopadhyay C, Dutta D, Chandran B. Interactions between exosomes from breast cancer cells and primary mammary epithelial cells leads to generation of reactive oxygen species which induce DNA damage response, stabilization of p53 and autophagy in epithelial cells. PLoS One 2014;9:e97580.

21. Pan J, Ding M, Xu K, Yang C, Mao LJ. Exosomes in diagnosis and therapy of prostate cancer. Oncotarget 2017;8:97693-700.

22. Zhang X, Yuan X, Shi H, Wu L, Qian H, et al. Exosomes in cancer: small particle, big player. J Hematol Oncol 2015;8:83.

23. Adem B, Vieira PF, Melo SA. Decoding the biology of exosomes in metastasis. Trends Cancer 2020;6:20-30.

24. Simpson RJ, Lim JW, Moritz RL, Mathivanan S. Exosomes: proteomic insights and diagnostic potential. Expert Rev Proteomics 2009;6:267-83.

25. Franzen CA, Blackwell RH, Foreman KE, Kuo PC, Flanigan RC, et al. Urinary exosomes: the potential for biomarker utility, intercellular signaling and therapeutics in urological malignancy. J Urol 2016;195:1331-9.

26. Panfoli I. Cancer exosomes in urine: a promising biomarker source. Transl Cancer Res 2017;6:S1389-93.

27. Gámez-Valero A, Lozano-Ramos SI, Bancu I, Lauzurica-Valdemoros R, Borràs FE. Urinary extracellular vesicles as source of biomarkers in kidney diseases. Front Immunol 2015;6:6.

28. Siravegna G, Marsoni S, Siena S, Bardelli A. Integrating liquid biopsies into the management of cancer. Nat Rev Clin Oncol 2017;14:531-48.

29. Di Meo A, Bartlett J, Cheng Y, Pasic MD, Yousef GM. Liquid biopsy: a step forward towards precision medicine in urologic malignancies. Mol Cancer 2017;16:80.

30. Gilligan KE, Dwyer RM. Engineering exosomes for cancer therapy. Int J Mol Sci 2017;18:1122.

31. Pitt JM, Charrier M, Viaud S, André F, Besse B, et al. Dendritic cell-derived exosomes as immunotherapies in the fight against cancer. J Immunol 2014;193:1006-11.

32. Liu C, Su C. Design strategies and application progress of therapeutic exosomes. Theranostics 2019;9:1015-28.

33. Peinado H, Alečković M, Lavotshkin S, Matei I, Costa-Silva B, et al. Melanoma exosomes educate bone marrow progenitor cells toward a pro-metastatic phenotype through MET. Nat Med 2012;18:883-91.

34. de Vrij J, Maas SL, Kwappenberg KM, Schnoor R, Kleijn A, et al. Glioblastoma-derived extracellular vesicles modify the phenotype of monocytic cells. Int J Cancer 2015;137:1630-42.

35. Kanada M, Bachmann MH, Hardy JW, Frimannson DO, Bronsart L, et al. Differential fates of biomolecules delivered to target cells via extracellular vesicles. Proc Natl Acad Sci U S A 2015;112:E1433-42.

36. Prunotto M, Farina A, Lane L, Pernin A, Schifferli J, et al. Proteomic analysis of podocyte exosome-enriched fraction from normal human urine. J Proteomics 2013;82:193-229.

37. Conde-Vancells J, Rodriguez-Suarez E, Embade N, Gil D, Matthiesen R, et al. Characterization and comprehensive proteome profiling of exosomes secreted by hepatocytes. J Proteome Res 2008;7:5157-66.

38. Zhang J, Li S, Li L, Li M, Guo C, et al. Exosome and exosomal microRNA: trafficking, sorting, and function. Genomics Proteomics Bioinformatics 2015;13:17-24.

39. Doyle LM, Wang MZ. Overview of extracellular vesicles, their origin, composition, purpose, and methods for exosome isolation and analysis. Cells 2019;8:727.

40. Li P, Kaslan M, Lee SH, Yao J, Gao Z. Progress in exosome isolation techniques. Theranostics 2017;7:789-804.

41. Yan Z, Dutta S, Liu Z, Yu X, Mesgarzadeh N, et al. A label-free platform for identification of exosomes from different sources. ACS Sens 2019;4:488-97.

42. Hurwitz SN, Sun L, Cole KY, Ford CR 3rd, Olcese JM, et al. An optimized method for enrichment of whole brain-derived extracellular vesicles reveals insight into neurodegenerative processes in a mouse model of Alzheimer's disease. J Neurosci Methods 2018;307:210-20.

43. Liu F, Vermesh O, Mani V, Ge TJ, Madsen SJ, et al. The exosome total isolation chip. ACS Nano 2017;11:10712-23.

44. Wu M, Ouyang Y, Wang Z, Zhang R, Huang PH, et al. Isolation of exosomes from whole blood by integrating acoustics and microfluidics. 
Proc Natl Acad Sci U S A 2017;114:10584-9.

45. Henderson MC, Azorsa DO. The genomic and proteomic content of cancer cell-derived exosomes. Front Oncol 2012;2:38.

46. Xiao H, Wong DT. Proteomic analysis of microvesicles in human saliva by gel electrophoresis with liquid chromatography-mass spectrometry. Anal Chim Acta 2012;723:61-7.

47. Jayachandran A, Manda SV, Shrestha R, Bridle KR, Prithviraj P, et al. Exosome-based liquid biopsy in the management of hepatocellular carcinoma. HR 2018;4:44.

48. Quandt D, Dieter Zucht H, Amann A, Wulf-Goldenberg A, Borrebaeck C, et al. Implementing liquid biopsies into clinical decision making for cancer immunotherapy. Oncotarget 2017;8:48507-20.

49. Kim DK, Lee J, Simpson RJ, Lötvall J, Gho YS. EVpedia: a community web resource for prokaryotic and eukaryotic extracellular vesicles research. Semin Cell Dev Biol 2015;40:4-7.

50. Skotland T, Ekroos K, Kauhanen D, Simolin H, Seierstad T, et al. Molecular lipid species in urinary exosomes as potential prostate cancer biomarkers. Eur J Cancer 2017;70:122-32.

51. Melo SA, Luecke LB, Kahlert C, Fernandez AF, Gammon ST, et al. Glypican-1 identifies cancer exosomes and detects early pancreatic cancer. Nature 2015;523:177-82.

52. Wang X, Luo G, Zhang K, Cao J, Huang C, et al. Hypoxic tumor-derived exosomal miR-301a mediates M2 macrophage polarization via PTEN/PI3K $\gamma$ to promote pancreatic cancer metastasis. Cancer Res 2018;78:4586-98.

53. Bastos N, Ruivo CF, da Silva S, Melo SA. Exosomes in cancer: use them or target them? Semin Cell Dev Biol 2018;78:13-21.

54. Pullan JE, Confeld MI, Osborn JK, Kim J, Sarkar K, et al. Exosomes as drug carriers for cancer therapy. Mol Pharm 2019;16:1789-98

55. Kanchanapally R, Deshmukh SK, Chavva SR, Tyagi N, Srivastava SK, et al. Drug-loaded exosomal preparations from different cell types exhibit distinctive loading capability, yield, and antitumor efficacies: a comparative analysis. Int J Nanomedicine 2019;14:531-41.

56. Luan X, Sansanaphongpricha K, Myers I, Chen H, Yuan H, et al. Engineering exosomes as refined biological nanoplatforms for drug delivery. Acta Pharmacol Sin 2017;38:754-63.

57. Sato YT, Umezaki K, Sawada S, Mukai SA, Sasaki Y, et al. Engineering hybrid exosomes by membrane fusion with liposomes. Sci Rep 2016;6:21933.

58. Vakhshiteh F, Atyabi F, Ostad SN. Mesenchymal stem cell exosomes: a two-edged sword in cancer therapy. Int J Nanomedicine 2019;14:2847-59.

59. Ueda N, Atsuta I, Ayukawa Y, Yamaza T, Furuhashi A, et al. Novel application method for mesenchymal stem cell therapy utilizing its attractant-responsive accumulation property. Appl Sci 2019;9:4908.

60. Harjunpää H, Llort Asens M, Guenther C, Fagerholm SC. Cell adhesion molecules and their roles and regulation in the immune and tumor microenvironment. Front Immunol 2019;10:1078.

61. Chen G, Huang AC, Zhang W, Zhang G, Wu M, et al. Exosomal PD-L1 contributes to immunosuppression and is associated with antiPD-1 response. Nature 2018;560:382-6.

62. Bellavia D, Raimondo S, Calabrese G, Forte S, Cristaldi M, et al. Interleukin 3- receptor targeted exosomes inhibit in vitro and in vivo chronic myelogenous leukemia cell growth. Theranostics 2017;7:1333-45.

63. Kim MS, Haney MJ, Zhao Y, Mahajan V, Deygen I, et al. Development of exosome-encapsulated paclitaxel to overcome MDR in cancer cells. Nanomedicine 2016;12:655-64.

64. Bovy N, Blomme B, Frères P, Dederen S, Nivelles O, et al. Endothelial exosomes contribute to the antitumor response during breast cancer neoadjuvant chemotherapy via microRNA transfer. Oncotarget 2015;6:10253-66.

65. Sun Z, Shi K, Yang S, Liu J, Zhou Q, et al. Effect of exosomal miRNA on cancer biology and clinical applications. Mol Cancer 2018;17:147.

66. Soung YH, Ford S, Zhang V, Chung J. Exosomes in cancer diagnostics. Cancers (Basel) 2017;9:8.

67. Rauschenberger L, Staar D, Thom K, Scharf C, Venz S, et al. Exosomal particles secreted by prostate cancer cells are potent mRNA and protein vehicles for the interference of tumor and tumor environment. Prostate 2016;76:409-24.

68. Van Deun J, Mestdagh P, Agostinis P, Akay Ö, Anand S, et al; EV-TRACK Consortium. EV-TRACK: transparent reporting and centralizing knowledge in extracellular vesicle research. Nat Methods 2017;14:228-32. 